\title{
Padrão de consumo de alimentos no Brasil
}

\author{
Alexandre Bragança Coelho' \\ Danilo Rolim Dias de Aguiar ${ }^{2}$ \\ Elaine Aparecida Fernandes ${ }^{3}$
}

Resumo: A aquisição de alimentos no Brasil tem sofrido modificações importantes nas últimas décadas. Fatores como urbanização, composição etária e outras transformações estruturais influenciam o montante despendido e a composição da cesta de alimentos consumida por uma família. Diante deste quadro de mudanças, é importante conhecer o novo padrão de consumo das famílias, em especial os determinantes da decisão de aquisição de alimentos. $\mathrm{O}$ objetivo deste estudo foi investigar o padrão de consumo de alimentos das famílias brasileiras, por meio da estimação de um modelo com variáveis de resposta qualitativa, incluindo dezoito tipos de alimentos, com base nos dados da Pesquisa de Orçamentos Familiares (POF) realizada em 2002/2003 pelo IBGE. O modelo escolhido para a estimação foi o Probit. Os resultados da estimação indicaram que a probabilidade de aquisição dos produtos básicos variou negativamente com a renda mensal familiar, enquanto as carnes, leite e demais produtos mostraram influência positiva da renda. As variáveis regionais também foram importantes na explicação da aquisição de diversos produtos, assim como as variáveis educacionais e as diferenças entre o meio rural e urbano. Domicílios chefiados por mulheres têm menor probabilidade de aquisição para consumo domiciliar de quase todos os produtos da cesta escolhida.

Palavras-chaves: Aquisição de alimentos; Pesquisa de Orçamentos Familiares (POF); modelo Probit.

\footnotetext{
${ }^{1}$ Professor do Departamento de Economia Rural da Universidade Federal de Viçosa (UFV). E-mail: acoelho@ufv.br

${ }^{2}$ Professor da Universidade Federal de São Carlos (Ufscar), Campus de Sorocaba. E-mail: danilo@ufscar.br.

${ }^{3}$ Professora do Departamento de Economia da Universidade Federal de Viçosa (UFV). E-mail: eafernandes@ufv.br
} 


\begin{abstract}
Food purchasing in Brazil has undergone major changes in the last few decades caused by structural shifts such as urbanization, changes in demographic characteristics and increase in participation of women in the labor force. Therefore, it is necessary to identify thoroughly new consumption patterns of families, especially concerning determinants of food purchasing decision. The objective of this study was to understand the consumption pattern of Brazilian families for food products by means of a qualitative response variable model including eighteen food products using POF 2002/2003 data. The Probit model was chosen to estimate this decision. Estimation results showed that purchasing probabilities of staple foods were negative related to family monthly income, while meat, milk and other products showed a positive relation. Regional, educational and urbanization variables were also important. Households whose head is a woman have smaller purchasing probabilities in most commodities in the sample.
\end{abstract}

Key-words: Food Purchase, Brazilian Household Budget Survey (POF), Probit Model.

Classificação JEL: D12, C25, R22.

\title{
1. Introdução
}

A aquisição de alimentos no Brasil tem sofrido modificações importantes nas últimas décadas. Fatores como urbanização, composição etária, presença de mulheres na força de trabalho e outras transformações estruturais influenciam o montante despendido e a composição da cesta de alimentos consumida por uma família, impondo novos desafios aos produtores rurais, à agroindústria, ao setor de distribuição de alimentos e ao governo. Além disso, o aumento, ainda que tímido, do grau de instrução escolar e da renda per capita potencializam estas transformações, visto que aumentam a consciência sobre o consumo de alimentos nutritivos e com menores riscos à saúde. Nesse cenário, é importante conhecer o novo padrão de consumo das famílias, em especial os determinantes da decisão de aquisição de alimentos.

O gasto com alimentação, apesar de perder importância nas últimas décadas, ainda é o segundo mais importante na participação das despesas das famílias, com 20,75\% do total (Tabela 1). Além disso, o item alimentação é o de maior peso no orçamento para famílias da zona rural e para as de baixa renda, notadamente nas regiões Norte e Nordeste do País. Segundo a Pesquisa de Orçamentos Familiares (POF) de 2002/2003, do Instituto Brasileiro de Geografia e Estatística (IBGE), para famílias de baixa renda (até dois salários mínimos mensais), estes 
gastos ainda representam $32,7 \%$ do total, ou seja, praticamente um terço das despesas totais dessas famílias (IBGE, 2004a).

Tabela 1. Participação na despesa de consumo monetária e não-monetária média mensal familiar, por situação do domicilio no ENDEF e na POF, segundo o tipo de despesa - Brasil - 1974/2003

\begin{tabular}{|c|c|c|c|c|c|c|}
\hline \multirow{5}{*}{$\begin{array}{l}\text { Tipos de } \\
\text { despesa }\end{array}$} & \multicolumn{6}{|c|}{$\begin{array}{l}\text { Participação na despesa de consumo monetária } \\
\text { e não-monetária média mensal familiar }\end{array}$} \\
\hline & \multirow{2}{*}{\multicolumn{2}{|c|}{ Total }} & \multicolumn{4}{|c|}{ Situação do domicílio } \\
\hline & & & \multicolumn{2}{|c|}{ Urbana } & \multicolumn{2}{|c|}{ Rural } \\
\hline & ENDEF(1) $^{(1)}$ & $\operatorname{POF}^{(2)}$ & ENDEF & POF & ENDEF & POF \\
\hline & 1974-1975 & 2002-2003 & 1974-1975 & 2002-2003 & $1974-1975^{(3)}$ & 2002-2003 \\
\hline Alimentação & 33,91 & 20,75 & 30,13 & 19,58 & 53,24 & 34,12 \\
\hline Habitação & 30,41 & 35,50 & 32,65 & 36,11 & 17,84 & 28,86 \\
\hline Transportes & 11,23 & 18,44 & 11,93 & 18,49 & 7,50 & 17,88 \\
\hline Assistência à saúde & 4,22 & 6,49 & 4,05 & 6,59 & 5,03 & 5,39 \\
\hline Educação & 2,28 & 4,08 & 2,58 & 4,32 & 0,87 & 1,46 \\
\hline Outros & 17,95 & 14,74 & 18,66 & 14,91 & 15,52 & 12,49 \\
\hline
\end{tabular}

Fonte: POF (2004b).

(1) Estudo Nacional de Despesas Familiares, realizado pelo Instituto Brasileiro de Geografia e Estatística (IBGE).

(2) Pesquisa de Orçamentos Familiares, realizada pelo Instituto Brasileiro de Geografia e Estatística (IBGE).

(3) Com exceção das áreas rurais das regiões Norte e Centro-Oeste.

Tem sido observada, nas últimas décadas, uma diminuição do consumo domiciliar per capita de determinados alimentos tradicionais [arroz, feijão $\mathrm{e}^{4}$ farinha de mandioca], e crescimento de outros, tais como frutas e alimentos preparados (Tabela 2). Os determinantes deste processo ainda necessitam ser estudados de forma mais detalhada.

Haines et al. (1988) argumentam que a decisão de consumo de produtos alimentares deve ser modelada como um problema de dois estágios. Isso ocorre porque a decisão de o que adquirir se processa de forma separada do quanto adquirir e os determinantes de cada decisão também podem ser diferentes. Muitos estudos procuraram estimar elasticidades-renda e elasticidades-preço da demanda para alimentos no Brasil, mas não os determinantes da decisão de aquisição. Há uma série de estudos ${ }^{5}$ que estimam elasticidades para um único produto ou para uma categoria de produtos (carnes, laticínios etc.).

\footnotetext{
$\overline{{ }^{4} \text { Ver Hoffmann }}$ (1995).

${ }^{5}$ Ver, por exemplo, Brandt et al. (1973), Fiallos (1982) e Bacchi (1989).
} 
Tabela 2. Quantidade anual per capita de alimentos adquiridos para consumo no domicilio $^{(1)}$ - Brasil - 1974/1996

\begin{tabular}{|c|c|c|c|c|}
\hline \multirow[t]{3}{*}{ Produtos selecionados } & \multicolumn{4}{|c|}{$\begin{array}{l}\text { Quantidade anual per capita de alimentos adquiridos } \\
\text { para consumo no domicilio }(\mathrm{kg})\end{array}$} \\
\hline & ENDEF & POF & POF & POF \\
\hline & 1974-1975 & 1987-1988 & 1995-1996 & $2002-2003$ \\
\hline Arroz Polido & 31,571 & 29,725 & 26,483 & 17,110 \\
\hline Feijão & 14,698 & 12,134 & 10,189 & 9,220 \\
\hline Batata-inglesa & 13,415 & 13,114 & 9,218 & 5,468 \\
\hline Farinha de mandioca & 5,207 & 4,679 & 3,765 & 3,313 \\
\hline Macarrão & 5,205 & 4,274 & 4,084 & 4,251 \\
\hline Carne Bovina & 16,161 & 18,509 & 20,800 & 14,574 \\
\hline Frango & 24,249 & 22,837 & 22,679 & 14,190 \\
\hline Leite de vaca pasteurizado (2) & 40,015 & 62,435 & 51,360 & 38,035 \\
\hline Alimentos preparados & 1,706 & 1,376 & 2,718 & 5,398 \\
\hline
\end{tabular}

Outros trabalhos ${ }^{6}$ procuraram estimar elasticidades para vários produtos ao mesmo tempo. Entre eles, pode-se destacar o trabalho de Hoffman (2000), que usa dados da POF 1995/96 do IBGE e o modelo poligonal com três segmentos para estimar elasticidades-renda para vários produtos alimentares. Já Aguero e Gould (2003) usam dados da POF 1995/96, procurando construir escalas de equivalência para comparar padrões de consumo para famílias de diferentes composições, usando dados agregados para produtos. Porém, apesar de existirem estudos que forneçam estimativas de elasticidades da demanda para vários alimentos, nota-se uma carência de trabalhos que modelem a decisão anterior de comprar ou não determinado produto, conforme o presente trabalho se propõe a fazer.

Outro ponto importante é a escolha do tipo de dados a serem utilizados nos estudos de demanda de alimentos. A maior parte dos estudos utiliza séries temporais, seja pela maior disponibilidade, seja pela facilidade de utilização. Entretanto, a maior parte dos dados de séries temporais é composta por dados agregados e há uma série de condições que devem ser observadas para que a teoria da demanda seja aplicável na forma agregada, da mesma maneira que na forma individual (Deaton e Muellbauer, 1980). Este estudo utilizou dados de seção cruzada, baseados nos questionários da POF de 2002/2003 do IBGE.

Assim, o objetivo desse trabalho é identificar o padrão de consumo de alimentos dos consumidores brasileiros. Especificamente, procurar-se-á identificar

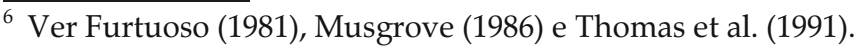


como o consumo de alimentos é afetado pelas seguintes variáveis: diferenças regionais; disparidades entre meio urbano e rural; níveis de escolaridade; raça; e outras medidas de heterogeneidade entre os consumidores brasileiros.

Os resultados deste artigo mostram que as variáveis regionais são muito importantes na explicação da aquisição de diversos produtos, assim como as variáveis educacionais e as diferenças entre os meios rural e urbano. Dessa forma, a ausência de variáveis como estas em modelos explicativos de padrões alimentares representam uma importante falha de especificação que pode comprometer os resultados e conclusões. Além disso, este artigo demonstra que a presença de mulheres na força de trabalho influencia decisivamente o padrão de alimentação domiciliar, pois domićlios chefiados por mulheres têm menor probabilidade de aquisição para consumo domiciliar de quase todos os produtos da cesta escolhida.

$\mathrm{O}$ artigo está organizado em cinco seções, incluindo esta introdução. $\mathrm{Na}$ seção dois, são descritos os dados utilizados no artigo. Na seção três, é apresentada a metodologia utilizada e, na seção quatro, os resultados das regressões, bem como sua discussão. Finalmente, na ultima seção, são apresentadas as conclusões do trabalho e algumas considerações finais.

\section{Dados utilizados}

Neste estudo, os dados utilizados para estimação do sistema de demanda são originários dos microdados ${ }^{7}$ da Pesquisa de Orçamentos Familiares realizada nos anos de 2002 e 2003 (POF 2002/2003) pelo IBGE. A POF 2002/2003 apresenta algumas diferenças importantes em relação às pesquisas anteriores. Em primeiro lugar, a pesquisa foi realizada em todo o território nacional, incluindo as áreas rurais de todas as regiões do País. Além disso, pela primeira vez, foram consideradas as aquisições não-monetárias ${ }^{8}$ na pesquisa, muito importantes especialmente nas áreas rurais.

A opção desta pesquisa é a de trabalhar com categorias de produtos bastante desagregadas, de forma a permitir a descrição mais precisa das escolhas dos consumidores frente à mudança nas variáveis escolhidas. Assim, no universo de produtos alimentares pesquisados, foram selecionados 18 produtos, pela sua importância no orçamento dos consumidores ou pelas relações de substituibilidade entre eles. Os produtos selecionados foram: açúcar, arroz, banana, batata, carne bovina de primeira, carne bovina de segunda, farinha de mandioca, feijão, carne de frango, leite em pó, leite fluido, macarrão, manteiga, margarina, pão francês, carne suína, queijos e tomate.

\footnotetext{
Microdados são os dados originais individuais retirados dos questionários da Pesquisa de Orçamentos Familiares (POF). Ver IBGE (2004c).

${ }^{8}$ Segundo o IBGE (2004a), "Despesas não-monetárias correspondem a tudo que é produzido, pescado, caçado, coletado ou recebido em bens utilizados ou consumidos durante o período de referência da pesquisa eque, pelo menos na ultima transação, não tenham passado pelo mercado".
} 
O tamanho da amostra da POF foi de 48.470 domicílios. Depois de alguns ajustes, em que foram eliminados todos os domicílios que não apresentavam informação de rendimento (renda zero) e os outliers ${ }^{9}$, a amostra usada neste estudo foi de 43.922 observações.

\section{Metodologia}

A metodologia utilizada para modelar as decisões de aquisição de alimentos foi um modelo com variáveis de resposta qualitativa, especificamente o modelo Probit ${ }^{10}$. Este modelo permite:

a) Explicar a escolha feita por indivíduos ou firmas;

b) Verificar quais fatores são mais importantes no processo de decisão;

c) Saber quanto cada fator explica as probabilidades de escolha;

d) Prever a escolha, dadas as características dos indivíduos.

\subsection{Modelo Estimado}

Supondo uma variável contínua e não-observável $y_{i}^{*}$ (nível de utilidade, por exemplo) tal que (Griffiths et al., 1993):

$y_{i}^{*}=X_{i} \beta+\varepsilon_{i}$

em que $X_{i}=$ covariadas; $\beta=$ vetor de parâmetros; $\varepsilon_{i}=$ erro aleatório, $\operatorname{comE}\left(\varepsilon_{i}\right)=0$.

Observa-se uma variável discreta binária:

$y_{i}=1 \quad$ se $y_{i}^{*}>y_{i}^{L}$

$y_{i}=0 \quad$ se $y_{i}^{*} \leq y_{i}^{L}$

em que $y_{i}^{L}=$ valor limite que determina a ocorrência do fenômeno.

\footnotetext{
${ }^{9}$ Foi realizado um exame detalhado nos valores das quantidades e despesas para cada produto para identificar outliers que pudessem prejudicar a estimação do sistema de demanda. Exemplos são gastos semanais de milhares de reais em algum produto (maior do que a renda do domicilio) ou aquisições de centenas de quilos, obviamente causados por erros de medida. Foram identificadas, assim, 309 observações que foram descartadas.

${ }^{10}$ Uma questão que pode surgir em função do procedimento adotado é sobre a razão de se utilizar apenas o modelo Probit e não o Logit. A escolha do modelo Probit decorre de ser este artigo parte de uma pesquisa maior, em que foi estimado um sistema de demanda para os 18 produtos alimentares da amostra. Este sistema foi estimado por meio do Procedimento de Shonkwiler e Yen (1999), que utiliza dois estágios de estimação para lidar com a natureza censurada dos dados. O primeiro estágio consiste nas chamadas "equações de seleção", que examinam os determinantes da decisão do consumidor em consumir ou não um determinado produto. Neste estágio, é necessário utilizar o modelo Probit, não sendo possível sua substituição pelo Logit, pois isso prejudicaria o segundo estágio de estimação.
} 
Assim:

$P\left(y_{i}=1\right)=P\left(y_{i}^{*}>y_{i}^{L}\right)$
$P\left(y_{i}=0\right)=P\left(y_{i}^{*} \leq y_{i}^{L}\right)$

Considerando $y_{i}^{L}=0$, tem-se:

$P\left(y_{i}=1\right)=P\left(y_{i}^{*}>0\right)=P\left(X_{i} \beta+\varepsilon_{i}>0\right)=P\left(\varepsilon_{i}>-X_{i} \beta\right)$

$P\left(y_{i}=0\right)=P\left(y_{i}^{*} \leq 0\right)=P\left(X_{i} \beta+\varepsilon_{i} \leq 0\right)=P\left(\varepsilon_{i} \leq-X_{i} \beta\right)$

Sendo $\varepsilon_{i}$ uma variável aleatória com função de distribuição de probabilidade cumulativa $\mathrm{F}($.$) , tem-se:$

$$
\begin{aligned}
& P\left(y_{i}=0\right)=F\left(-X_{i} \beta\right) \\
& P\left(y_{i}=1\right)=1-F\left(-X_{i} \beta\right)
\end{aligned}
$$

Considerando-se $\mathrm{F}($.) simétrica, tem-se que:

$$
\begin{aligned}
& P\left(y_{i}=0\right)=1-F\left(X_{i} \beta\right) \equiv 1-P_{i} \\
& P\left(y_{i}=1\right)=F\left(X_{i} \beta\right) \equiv P_{i}
\end{aligned}
$$

Em que $P_{i}$ é a probabilidade de ocorrência do fenômeno (neste caso, da aquisição do alimento $i$ ). A definição de $F\left(\right.$.) assegura que $0 \leq P\left(y_{i}=1\right) \leq 1$, para todo $X_{i}$. Da posse da definição de $P\left(y_{i}=0\right)$ e $P\left(y_{i}=1\right)$, pode-se definir uma função de Verossimilhança $L$, que é a função de probabilidade conjunta das ocorrências $\left(y_{i}=1\right)$ e não-ocorrências $\left(y_{i}=0\right)$. Suponha $\mathrm{n}_{1}$ ocorrências e $\mathrm{n}_{2}$ não-ocorrências, de forma que $\mathrm{n}=\mathrm{n}_{1}+\mathrm{n}_{2}$. Tem-se que:

$$
L=P\left(y_{1}, y_{2}, \ldots, y_{n}\right)
$$

Dada a independência dos $y^{\prime} s$ :

$L=P\left(y_{1}\right) \cdot P\left(y_{2}\right) \cdot P\left(y_{3}\right) \ldots P\left(y_{n}\right)$

Ordenando pelas $\mathrm{n}_{1}$ ocorrências e $\mathrm{n}_{2}$ não-ocorrências:

$$
\begin{aligned}
& L=P\left(y_{1}\right) \cdot P\left(y_{2}\right) \ldots P\left(y_{n_{1}}\right) \cdot P\left(y_{n_{1}+1}\right) \ldots, P\left(y_{n}\right) \\
& L=P_{1} \cdot P_{2} \ldots P_{n_{1}}\left(1-P_{n_{1}+1}\right) \cdot\left(1-P_{n_{1}+1}\right) \ldots\left(1-P_{n}\right) \\
& L=\prod_{i=1}^{n_{1}} P_{i} \prod_{i=n_{1}+1}^{n_{1}}\left(1-P_{i}\right)=\prod_{i=1}^{n_{1}} P_{i}^{y_{i}}\left(1-P_{i}\right)^{1-y_{i}}
\end{aligned}
$$

Da definição de $P_{i}$ em (11):

$$
L=\prod_{i=1}^{n_{1}}\left[F\left(X_{i} \beta\right)\right]^{y_{i}}\left[1-F\left(X_{i} \beta\right)\right]^{1-y_{i}}
$$


Maximizar L é o mesmo que maximizar o logaritmo de L. Assim:

$$
\log L=\sum_{i=1}^{n} y_{i} \log \left[F\left(X_{i} \beta\right)\right]+\sum_{i=1}^{n}\left(1-y_{i}\right) \log \left[1-F\left(X_{i} \beta\right)\right]
$$

Os estimadores de Máxima Verossimilhança são os valores de $\beta$ que maximizam $\log$ L. No modelo Probit, a função de distribuição de probabilidade cumulativa $\mathrm{F}($.$) é a normal padronizada. Assim:$

$$
P_{i}=F\left(X_{i} \beta\right)=\int_{-\infty}^{X_{i} \beta} \frac{1}{2 \pi} e^{-\frac{z^{2}}{2}} d z \text {, em que } z \text { é a normal padronizada }
$$

\subsection{Efeitos marginais das variáveis}

Para se analisar a magnitude do efeito de cada variável na probabilidade de aquisição de cada produto alimentar, o exame dos coeficientes não é o mais indicado, pois eles não fornecem os efeitos marginais de cada variável na probabilidade. Para calcular os efeitos marginais, é necessário distinguir as variáveis contínuas e as variáveis binárias. No primeiro caso, o efeito marginal é calculado da seguinte forma (tomando como exemplo a variável renda):

$$
E M_{\text {renda }}=f\left(X_{i} \beta\right) \cdot \beta_{\text {renda }}
$$

em que $E M_{\text {renda }}=$ efeito marginal da variável renda; $f\left(X_{i} \beta\right)=$ função de densidade de probabilidade da normal padrão avaliada no ponto $I_{i}=X_{i} \beta ; \beta_{\text {renda }}$ = coeficiente da variável renda.

Os efeitos marginais serão calculados na média da amostra. No caso das variáveis binárias, o efeito marginal será calculado da seguinte forma:

$$
E M_{x_{k}}=P\left[\left(y_{i}=1 / x_{k}=1\right)\right]-P\left[\left(y_{i}=1 / x_{k}=0\right)\right]
$$

em que $E M_{x_{k}}=$ efeito marginal da variável binária $x_{k} ; P\left[\left(y_{i}=1 / x_{k}=1\right)\right]=$ probabilidade de aquisição do produto quando $x_{k}=1 ; P\left[\left(y_{i}=1 / x_{k}=0\right)\right]=$ probabilidade de aquisição do produto quando $x_{k}=0$.

\section{Resultados e Discussão}

\subsection{Estimativas do Modelo}

As variáveis explicativas utilizadas e suas denominações estão descritas no Quadro 1. 
Quadro 1. Variáveis explicativas utilizadas na estimação

\section{Variáveis explicativas:}

- Constante (chamada de ONE)

- Logaritmo natural da renda mensal familiar (chamada de RENDA);

- Dummy regional:

- Norte (chamada de NORTE);

- Nordeste (chamada de NORDESTE);

- Sul (chamada de SUL);

- Sudeste (será o default);

- Centro-Oeste (chamada de CENTRO-O);

- Dummy de situação de domicílio (chamada de URBANO):

- Urbano = 1;

- Rural $=0$.

- Dummy de educação do responsável pelo domicilio:

- Analfabeto (chamada de ANALFA)

- Primeiro grau incompleto (chamada de PRIINC);

- Primeiro grau completo (será o default);

- Segundo grau incompleto (chamada de SEGINC);

- Segundo grau completo (chamada de SEGCOMP);

- Superior incompleto (chamada de SUPINC);

- Superior completo (chamada de SUPCOMP);

- Dummy de raça do responsável pelo domicilio:

- Negra (chamada de NEGRA);

- Branca (será o default);

- Amarela (chamada de AMARELA);

- Parda (chamada de PARDA);

- Outra (chamada de OUTRA);

- Dummy captando se a responsável pelo domicílio é mulher (chamada de MULHER):

- Mulher =1;

- Homem $=0$

- Dummy captando se o domicílio tem empregada doméstica (chamada de DOMESTIC):

- Possui empregada $=1$;

- Não possui empregada $=0$;

- Dummy captando se o domicílio tem geladeira (chamada de REFRIG):

- Possui geladeira $=1$;

- Não possui geladeira $=0$; 
Os resultados para cada produto estão descritos na Tabela $3^{11}$. Pode-se notar que 263 dos 360 coeficientes são significativos, ou $73,06 \%$ do total ${ }^{12}$. Os sinais das variáveis, em sua maioria, estão também de acordo com o esperado. No caso da renda, o aumento pode estar relacionado a uma menor probabilidade de aquisição para arroz e açúcar. Para feijão e farinha de mandioca, os coeficientes da renda também são negativos, porém, não-significativos estatisticamente. Nos demais produtos, o aumento da renda causa aumento na probabilidade de aquisição do produto.

Em relação às variáveis que captam as diferenças regionais, a maior parte dos coeficientes é significativo, indicando que há diferenças regionais em relação à região Sudeste (default), mesmo controlando-se para as demais variáveis. Este é um resultado importante, visto que indica que a probabilidade de aquisição de determinado produto é influenciada por fatores regionais, independente das conhecidas disparidades regionais de renda. Por exemplo, a probabilidade de consumo de carne suína é muito maior no Sul do que nas demais regiões do País. Da mesma forma, as variáveis "Norte" e "Nordeste" têm influência positiva na probabilidade de aquisição de produtos básicos, como arroz, feijão, farinha de mandioca, além de leite em pó e margarina. A variável "Sudeste" influencia positivamente a probabilidade de consumo de pão francês e a variável "Centro-Oeste" tem efeito positivo para arroz e leite fluido. Alguns resultados são surpreendentes, como o coeficiente positivo para o Nordeste no caso de carne bovina de primeira, um resultado não esperado dado que o consumo médio nessa região é menor do que no Sudeste ${ }^{13}$. Entretanto, como o efeito da renda é positivo para esse produto, a conclusão parece ser que, controlado para a renda, $\mathrm{o}$ consumidor do Nordeste tem maior probabilidade de adquirir, para o consumo domiciliar, carne bovina de primeira do que o consumidor do Sudeste. A maior freqüência de alimentação fora do domicílio no Sudeste pode explicar também parte deste resultado.

\footnotetext{
${ }^{11} \mathrm{O}$ software utilizado para a estimação foi o GAUSS 6.0 for Windows, Copyright 1984-2003, Aptech Systems.

${ }^{12}$ Foram realizados testes de heterocedasticidade nos resíduos das 18 regressões, utilizando-se o teste LM para heterocedasticidade com o método de regressão artificial descrito em Davidson e MacKinnon (1993, p. 523-528). Os resultados mostraram problemas de heterocedasticidade em 12 das 18 regressões estimadas (as que não apresentaram problemas foram: feijão, leite em pó, macarrão, manteiga, pão francês e suínos). Entretanto, Greene (2000, p. 829) argumenta que Davidson e Mackinnon realizaram experimentos de Monte Carlo para examinar o teste acima e concluíram que, apesar de ele ser adequado para testar variáveis omitidas no modelo probit, seu desempenho para teste de heterocedasticidade é problemático. Assim, preferiu-se deixar a regressão dessa forma, o que pode ser entendido como uma limitação deste estudo.

${ }^{13}$ Segundo a POF 2002/2003 (IBGE, 2004a), o consumo domiciliar per capita anual de carne bovina de primeira é de 6,253 kg no Sudeste e 4,630 kg no Nordeste.
} 
No caso das variáveis que procuram captar o efeito do nível educacional do responsável pelo domicílio na probabilidade de aquisição, grande parte dos coeficientes das variáveis "segundo grau incompleto" e "segundo grau completo" não é significativo. Isso indica que há pouca diferença na probabilidade de aquisição para domicílios com responsáveis com primeiro grau completo e segundo grau, completo ou não. Entretanto, os coeficientes para as variáveis "analfabeto", "superior incompleto" e "superior completo" são, na maior parte, significativos, sendo que os sinais são, na grande parte, negativos. No caso de responsáveis analfabetos, são observados valores positivos para açúcar, arroz, farinha de mandioca, feijão e carne bovina de segunda (este último, com coeficiente não-significativo) e negativos para os demais produtos. No caso de variáveis indicativas de curso superior, com exceção de queijos e manteiga (este último tem coeficiente não-significativo), todos os coeficientes são negativos, indicando menores probabilidades de aquisição na comparação com lares chefiados por pessoas com apenas o primeiro grau completo. A explicação para esse resultado pode estar na menor proporção de refeições realizadas no domicílio para aqueles com curso superior, que causaria naturalmente uma menor probabilidade de aquisição para consumo domiciliar. Além disso, a demanda por alimentos energéticos (arroz, açúcar, farinha de mandioca, etc.) tende a ser maior para aqueles que executam trabalhos manuais, como os analfabetos e aqueles com primeiro grau incompleto, em relação aos trabalhadores com maior escolaridade.

No caso da variável que capta as diferenças entre o meio urbano e rural, os coeficientes não foram significativos apenas para o caso do frango, macarrão e carne suína. Em relação aos sinais dos coeficientes, os resultados foram de acordo com o esperado, com valores negativos para os produtos mais energéticos (açúcar, arroz, farinha de mandioca, etc.), indicando maior probabilidade de consumo na zona rural, e valores positivos para produtos como pão francês, carne bovina, queijos, leite em pó, entre outros, cuja probabilidade de consumo é maior no meio urbano. 
346 - Padrão de consumo de alimentos no Brasil

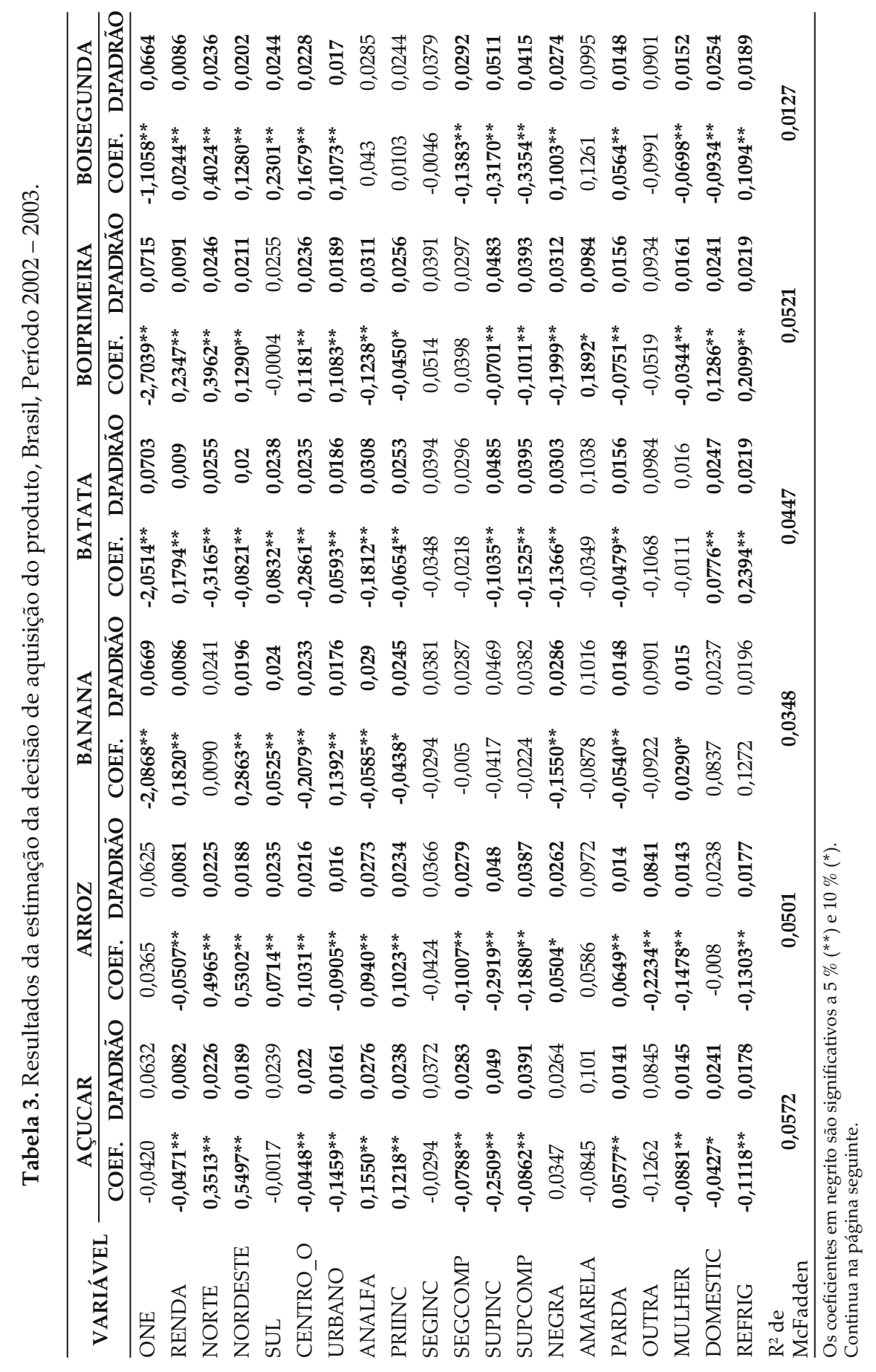

RESR, Piracicaba, SP, vol. 47, no 02, p. 335-362, abr/jun 2009 - Impressa em julho 2009 
Alexandre Bragança Coelho, Danilo Rolim Dias de Aguiar e Elaine Aparecida Fernandes 347

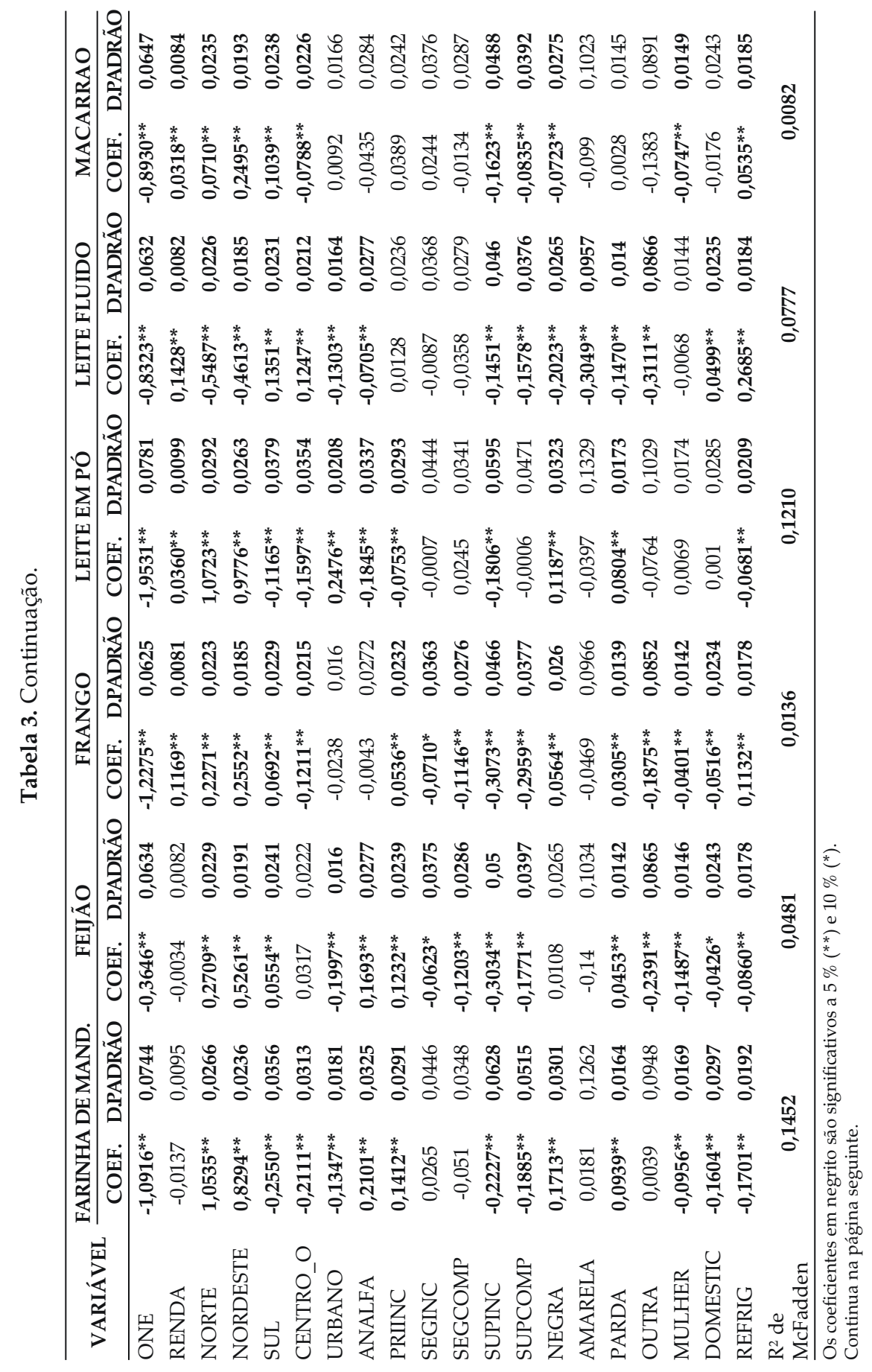

RESR, Piracicaba, SP, vol. 47, no 02, p. 335-362, abr/jun 2009 - Impressa em julho 2009 


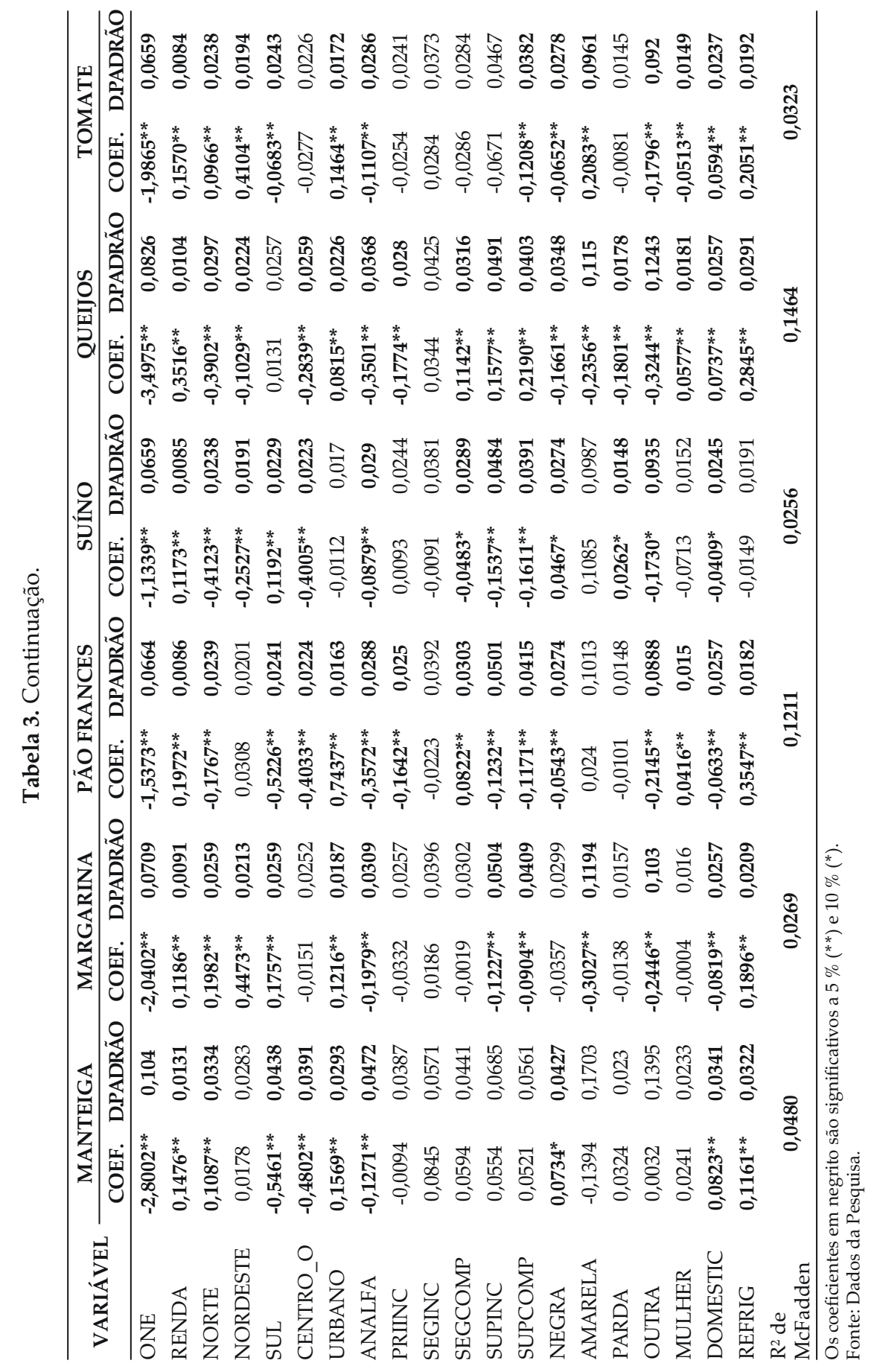

RESR, Piracicaba, SP, vol. 47, no 02, p. 335-362, abr/jun 2009 - Impressa em julho 2009 
No caso de variáveis que captam a influência da raça na probabilidade de aquisição, 30 dos 72 coeficientes não são significativos, especialmente no caso das variáveis "amarelo" e "outra". Dessa forma, na maior parte dos casos, a diferença entre os domicílios chefiados por indivíduos que se declararam amarelos ou de outras raças (que não brancos, negros ou pardos) e os domicílios chefiados por brancos foi insignificante. No caso de negros e pardos, parece haver uma influência positiva na probabilidade de aquisição de açúcar, arroz, carne bovina de segunda, farinha de mandioca, entre outras e negativa para leite fluido, carne bovina de primeira, banana, batata, tomate e queijos.

No caso da variável "mulher", que representa o fato de o responsável pelo domicílio ser do sexo feminino, a maior parte dos coeficientes é significativo e negativo, com destaque para as carnes em geral, açúcar, arroz e feijão. No caso de leite e derivados, a influência da mulher não é significativa, com exceção de Queijos, no qual ela exerce influência positiva. Nos casos da banana e pão francês, esta variável tem influência positiva na probabilidade de aquisição. É difícil identificar uma explicação para o comportamento desta variável. A hipótese de que as mulheres, com maior preocupação com a saúde do que os homens, possam determinar uma alimentação mais rica em frutas e verduras no domicílio, não pôde ser testada adequadamente, pela reduzida quantidade de produtos na cesta escolhida, ou seja, não se sabe se a menor probabilidade de consumo apresentada na maior parte dos produtos da cesta significa efetivamente uma escolha de outros produtos não incluídos, como frutas e verduras. $O$ fato é que domicílios chefiados por mulheres têm menor probabilidade de aquisição dos produtos da cesta.

As demais variáveis, "doméstica" e "geladeira", procuram identificar a influência da presença de empregada doméstica e refrigerador no domicílio, respectivamente, sobre a probabilidade de aquisição dos alimentos. No caso da variável "doméstica", os resultados foram o oposto do esperado. A presença de empregada doméstica no domicílio diminui a probabilidade de aquisição de feijão e carnes em geral, com exceção da carne bovina de primeira. Para o arroz, o coeficiente dessa variável não é significativo. Apenas para batata e tomate (e alguns outros produtos, como leite e derivados, cuja expectativa era nula), os valores foram positivos. A expectativa inicial era de que o fato de possuir empregada doméstica tornasse mais provável à realização de refeições no domicílio, já que representaria uma solução para a restrição de tempo no preparo das mesmas, pela presença de uma profissional responsável pela preparação $0^{14}$. A explicação pode estar no fato de que a distância entre trabalho e residência para a maior parte das pessoas impeça a realização de refeições no domicílio, mesmo para pessoas que possuam empregadas domésticas. A influência negativa decorreria, assim, da maior probabilidade de presença de empregadas em lares onde a restrição e custo do tempo (e distância) fossem maiores.

${ }^{14} \mathrm{Da}$ mesma forma, mesmo que a empregada doméstica não fosse responsável pelas refeições, a realização das demais tarefas domésticas permitiria maior tempo para o preparo das refeições pelo responsável. 
No caso da variável "geladeira", os resultados foram de acordo com o esperado, principalmente no caso do leite. A presença de geladeira aumenta a probabilidade de aquisição de leite fluido e diminui a probabilidade do leite em pó, um resultado importante para explicar a razão de famílias mais pobres geralmente consumirem um produto relativamente mais caro como o leite em pó na presença de outro com as mesmas propriedades e bem mais barato (leite fluido). A capacidade de conservação é um fator importante para essas famílias. A presença de geladeira no domicílio também tem efeito positivo na probabilidade de aquisição de carnes em geral, com exceção da carne suína, cujo efeito não é significativo. O efeito positivo também é observado para queijos. Já a influência negativa é observada para produtos básicos, como arroz, feijão, farinha de mandioca e açúcar. O que pode estar ocorrendo aí é que, controlando para as demais variáveis, a posse de geladeira permita aos domicílios substituir parte dos produtos básicos por aqueles acima citados que necessitam de refrigeração.

\subsection{Efeitos marginais}

Os resultados dos cálculos dos efeitos marginais estão nas Tabelas 4 a 11 . Pode-se notar pelas fórmulas dos efeitos marginais que os sinais são os mesmos da análise dos coeficientes do item anterior. Dessa forma, passa-se diretamente à análise das magnitudes para cada variável. No caso da renda (Tabela 4), os efeitos na probabilidade de aquisição são baixos. Em termos positivos, destaca-se o caso do queijo: um aumento de uma unidade no logaritmo da renda ${ }^{15}$ causa um aumento de 8,81 pontos percentuais (p.p.) na probabilidade de aquisição. Carne bovina de primeira (7,37 p.p.) e pão francês (6,99 p.p.) vêm logo em seguida. Em termos negativos, arroz (-1,99 p.p.) e açúcar (-1,82 p.p.) apresentam os menores valores.

Tabela 4. Efeitos marginais da variável Renda, Brasil, Período 2002 - 2003

\begin{tabular}{lc}
\hline PRODUTOS & EFEITO MARGINAL \\
\hline AÇÚCAR & $-0,0182$ \\
ARROZ & $-0,0199$ \\
BANANA & 0,0637 \\
BATATA & 0,0554 \\
BOIPRIMEIRA & 0,0737 \\
BOISEGUNDA & 0,0081 \\
FARINHA DE MANDIOCA & $-0,0036$ \\
FEIJÃO & $-0,0013$ \\
FRANGO & 0,0458 \\
LEITE EM PÓ & 0,0081 \\
LEITE FLUIDO & 0,0570 \\
MACARRÃO & 0,0111 \\
\hline
\end{tabular}

$\overline{{ }^{15} \mathrm{O} \text { que significa, }}$ no ponto médio, um aumento da renda familiar mensal de $\mathrm{R} \$ 1.525,63$ para $\mathrm{R} \$ 4.147,01$. 
Tabela 4. Continuação.

\begin{tabular}{lc}
\hline PRODUTOS & EFEITO MARGINAL \\
\hline MANTEIGA & 0,0164 \\
MARGARINA & 0,0351 \\
PAO FRANCÊS & 0,0699 \\
SUÍNO & 0,0405 \\
QUEIJOS & 0,0881 \\
TOMATE & 0,0566 \\
\hline
\end{tabular}

Fonte: Dados da Pesquisa.

Em relação aos efeitos marginais regionais (Tabela 5), as magnitudes são bem superiores às observadas para a renda. Entre os produtos básicos, o fato de o domicílio estar nas regiões Norte e Nordeste tem influência positiva na probabilidade de aquisição em relação à região Sudeste. Para o caso da farinha de mandioca, um domicílio na região Norte apresenta uma probabilidade de aquisição 29,92 pontos percentuais superior a um domicílio do Sudeste com as mesmas características. No caso do Nordeste, essa diferença é de 21,65 pontos percentuais. Para o feijão, o destaque é o Nordeste: a diferença em relação ao Sudeste chega a 19,76 pontos percentuais. Para o arroz, o consumidor do Norte e Nordeste tem uma probabilidade de adquirir o produto cerca de 20 pontos percentuais maior que no caso do consumidor do Sudeste.

Tabela 5. Efeitos marginais das variáveis Regionais, Brasil, Período 2002 - 2003

\begin{tabular}{lcccc}
\hline \multicolumn{1}{c}{ PRODUTOS } & NORTE & NORDESTE & SUL & CENTRO-OESTE \\
\hline AÇÚCAR & 0,1314 & 0,2102 & $-0,0006$ & $-0,0154$ \\
ARROZ & 0,1910 & 0,2044 & 0,0258 & 0,0375 \\
BANANA & 0,0030 & 0,1027 & 0,0178 & $-0,0647$ \\
BATATA & $-0,0944$ & $-0,0266$ & 0,0284 & $-0,0864$ \\
BOIPRIMEIRA & 0,1299 & 0,0387 & $-0,0001$ & 0,0353 \\
BOISEGUNDA & 0,1354 & 0,0396 & 0,0737 & 0,0527 \\
FARINHA DE MANDIOCA & 0,2992 & 0,2165 & $-0,0352$ & $-0,0301$ \\
FEIJÃO & 0,0977 & 0,1976 & 0,0190 & 0,0108 \\
FRANGO & 0,0887 & 0,0999 & 0,0266 & $-0,0451$ \\
LEITE EM PÓ & 0,2505 & 0,2180 & $-0,0123$ & $-0,0163$ \\
LEITE FLUIDO & $-0,2159$ & $-0,1824$ & 0,0516 & 0,0477 \\
MACARRÃO & 0,0237 & 0,0874 & 0,0351 & $-0,0251$ \\
MANTEIGA & 0,0158 & 0,0024 & $-0,0483$ & $-0,0446$ \\
MARGARINA & 0,0528 & 0,1318 & 0,0464 & $-0,0036$ \\
PAO FRANCÊS & $-0,0608$ & 0,0100 & $-0,1929$ & $-0,1460$ \\
SUÚNO & $-0,1418$ & $-0,0907$ & 0,0458 & $-0,1382$ \\
QUEIJOS & $-0,0927$ & $-0,0280$ & 0,0037 & $-0,0710$ \\
TOMATE & 0,0329 & 0,1499 & $-0,0221$ & $-0,0091$ \\
\hline
\end{tabular}

Fonte: Dados da Pesquisa. 
O Norte, surpreendentemente, também se destaca no consumo de carnes, especialmente a carne bovina. Em relação ao consumo de leite, Norte e Nordeste têm comportamento semelhante: um domicílio dessas regiões tem maior probabilidade de aquisição de leite em pó $(25,05$ p.p. e 21,80 p.p., respectivamente) e menor probabilidade de aquisição de leite fluido (-21,59 p.p.e $-18,24$ p.p., respectivamente) do que um domicílio com as mesmas características da região Sudeste. Esse comportamento é o inverso no Sul e Centro-Oeste, onde o efeito marginal é positivo para leite fluido e negativo para leite em pó. Outro destaque é o efeito marginal positivo do Nordeste no caso da banana. Isso talvez seja explicado pelo baixo preço dessa fruta em relação a outras, como a maçã ou a pêra, cujo consumo é mais difundido no Sul e Sudeste.

No caso do consumo carne suína, a probabilidade aumenta para as regiões Sul e Sudeste em comparação com as demais regiões do País. Para o pão francês, os destaques positivos são Nordeste e Sudeste e o negativo fica com a região Sul. Uma surpresa é a magnitude do efeito marginal para o Nordeste no caso do tomate e carnes bovinas de primeira e de segunda. Controlando para a renda e demais variáveis, um domicílio do Nordeste tem maior probabilidade de adquirir carne de primeira (3,87 p.p.), de segunda (3,96 p.p.) e tomate (14,99 p.p.) do que no Sudeste.

No Centro-Oeste, a probabilidade de aquisição é, na maior parte dos casos, inferior à região Sudeste, com destaque para o pão francês (-14,60 p.p.). A grande exceção é a carne de boi de segunda, com probabilidade 5,27 pontos percentuais maior do que no Sudeste. Para a região Sul, o destaque positivo, além da carne suína, fica com a carne bovina de segunda (7,37 p.p. maior) e margarina (4,64 p.p maior). Já o negativo fica com o pão francês (-19,29 p.p) e manteiga (-4,83 p.p.).

Em relação às diferenças entre meio urbano e rural (Tabela 6), destaca-se o efeito marginal no caso do pão francês. Um domicílio do meio urbano tem probabilidade 27,95 pontos percentuais maior de aquisição de pão francês que o mesmo domicílio na zona rural. Esse resultado era esperado, pela questão da proximidade de pontos de venda e hábitos alimentares diferentes. Outros destaques do consumo urbano são leite em pó, tomate, banana e carne bovina. $\mathrm{O}$ meio rural destaca-se nas probabilidades de aquisição dos produtos básicos, em especial feijão, açúcar e arroz. Outro destaque é o leite fluido, em que o meio rural tem probabilidade 5,19 pontos percentuais maior do que no meio urbano. $\mathrm{O}$ papel da produção própria e o autoconsumo certamente influenciam esse último resultado, já que o IBGE incluiu as aquisições não-monetárias no total adquirido. 
Tabela 6. Efeitos marginais da variável "urbana”, Brasil, Período 2002 - 2003

\begin{tabular}{lc}
\hline PRODUTOS & EFEITO MARGINAL \\
\hline AÇÚCAR & $-0,0567$ \\
ARROZ & $-0,0357$ \\
BANANA & 0,0477 \\
BATATA & 0,0181 \\
BOIPRIMEIRA & 0,0333 \\
BOISEGUNDA & 0,0348 \\
FARINHA DE MANDIOCA & $-0,0365$ \\
FEIJÃO & $-0,0768$ \\
FRANGO & $-0,0094$ \\
LEITE EM PÓ & 0,0515 \\
LEITE FLUIDO & $-0,0519$ \\
MACARRÃO & 0,0032 \\
MANTEIGA & 0,0162 \\
MARGARINA & 0,0350 \\
PAO FRANCÊS & 0,2795 \\
SUÍNO & $-0,0039$ \\
QUEIJOS & 0,0200 \\
TOMATE & 0,0518 \\
\hline
\end{tabular}

Fonte: Dados da Pesquisa

Em relação à educação do responsável pelo domicílio (Tabela 7), as magnitudes não são tão expressivas quanto no caso das variáveis regionais, mas parecem predominar em relação aos efeitos marginais da variável renda. No caso da variável "analfabeto" (responsável pelo domicílio analfabeto), destacam-se a menor probabilidade de aquisição de pão francês (-12,83 p.p.) e queijos $(-8,29$ p.p.) e o maior probabilidade de aquisição de feijão (6,46 p.p.) e açúcar (5,99 p.p.) em relação a domicílios chefiados por indivíduos que completaram o primeiro grau. Para a variável "primeiro grau incompleto" (responsável pelo domicílio com primeiro grau incompleto), o comportamento é semelhante a "analfabeto". As diferenças aparecem apenas para frango, leite fluido, macarrão e carne suína, que apresentam efeitos marginais positivos, ao contrário de "analfabeto".

As variáveis "segundo grau incompleto" (responsável pelo domicílio com segundo grau incompleto) e "segundo grau completo" (responsável pelo domicílio com segundo grau completo), como visto no item anterior, apresentam a maior parte dos seus coeficientes não-significativos estatisticamente. Para "segundo grau completo", entretanto, destacam-se os efeitos marginais para carne bovina de segunda (-4,46 p.p.), arroz (-3,88 p.p.) e queijos (3,33 p.p.). No 
caso da variável "curso superior incompleto" (responsável pelo domicílio com curso superior incompleto), destaque para a menor probabilidade de aquisição de frango (-11,58 p.p.) e arroz (-10,89 p.p.) e maior probabilidade para queijos $(4,68$ p.p.). De forma geral, predominam os efeitos marginais negativos para a cesta selecionada. O padrão se repete no caso de "curso superior completo" (responsável pelo domicílio curso superior completo). Os destaques neste caso são a menor probabilidade no caso de carne de frango (-11,17 p.p.) e carne bovina de segunda (-10,07 p.p.) e a maior probabilidade para queijos (6,65 p.p.). Aliás, nessas últimas duas categorias, os efeitos marginais são positivos apenas para queijos e manteiga, indicando que a probabilidade de aquisição é consistentemente menor, controlando para as outras variáveis, do que em domicílios com responsáveis com apenas o primeiro grau completo. Entre as razões para este resultado, a menor freqüência de refeições no domicílio para consumidores com maior escolaridade e o maior consumo de alimentos preparados certamente estão entre as mais importantes para a compreensão deste padrão de consumo.

No caso dos efeitos marginais para as variáveis indicativas da raça do responsável pelo domicílio (Tabela 8), destacam-se os impactos negativos da variável "negra" no consumo de leite fluido (-8,06 p.p.) e carne de boi de primeira (-6,05 p.p.) em relação a domicílios chefiados por indivíduos que se declararam brancos. Quanto aos efeitos positivos, destacam-se a farinha de mandioca $(4,60$ p.p.) e carne bovina de segunda (3,35 p.p.). Para a variável "parda", o padrão de efeitos marginais é semelhante, com destaque para leite fluido (-5,86 p.p.) e arroz (2,55 p.p.). Para a variável "amarela", a maior parte dos efeitos marginais se origina de coeficientes não-significativos; dos poucos resultados significativos, destacam-se a maior probabilidade de aquisição de tomate ( 7,84 p.p.) e de carne bovina de primeira (6,49 p.p.). O destaque negativo foi o leite fluido (-12,09 p.p.), com probabilidade de aquisição muito inferior do que domicílios chefiados por brancos. Para a variável "outra", todos os efeitos marginais significativos foram negativos. Destaque novamente para o leite fluido (-12,33 p.p.) e também o feijão (-8,56 p.p.). De forma geral, pode-se afirmar que as variáveis indicativas de raça parecem importantes na explicação dos padrões de aquisição dos consumidores, mesmo controlando para as demais variáveis. Elas refletem provavelmente heranças culturais diferentes e padrões de consumo passados de geração a geração e ainda refletem certo padrão de desigualdade, principalmente entre brancos e negros/pardos. 
Tabela 7. Efeitos marginais das variáveis de nível educacional do responsável pelo domicílio, Brasil, Período 2002 - 2003

\begin{tabular}{lcccccc}
\hline \multicolumn{1}{c}{ PRODUTOS } & ANALFA & PRIINC & SEGINC & SEGCOMP & SUPINC & SUPCOMP \\
\hline AÇÚCAR & 0,0599 & 0,0468 & $-0,0110$ & $-0,0294$ & $-0,0901$ & $-0,0321$ \\
ARROZ & 0,0370 & 0,0403 & $-0,0165$ & $-0,0388$ & $-0,1089$ & $-0,0715$ \\
BANANA & $-0,0206$ & $-0,0154$ & $-0,0104$ & $-0,0018$ & $-0,0147$ & $-0,0079$ \\
BATATA & $-0,0554$ & $-0,0208$ & $-0,0112$ & $-0,0070$ & $-0,0325$ & $-0,0471$ \\
BOIPRIMEIRA & $-0,0384$ & $-0,0143$ & 0,0169 & 0,0130 & $-0,0221$ & $-0,0316$ \\
BOISEGUNDA & 0,0147 & 0,0035 & $-0,0016$ & $-0,0446$ & $-0,0959$ & $-0,1007$ \\
FARINHA DE & 0,0559 & 0,0364 & 0,0065 & $-0,0120$ & $-0,0477$ & $-0,0412$ \\
MANDIOCA & 0,0646 & 0,0467 & $-0,0229$ & $-0,0436$ & $-0,1049$ & $-0,0633$ \\
FEIJÃO & $-0,0017$ & 0,0212 & $-0,0277$ & $-0,0445$ & $-0,1158$ & $-0,1117$ \\
FRANGO & $-0,0404$ & $-0,0175$ & $-0,0002$ & 0,0060 & $-0,0396$ & $-0,0001$ \\
LEITE EM PÓ & $-0,0281$ & 0,0051 & $-0,0035$ & $-0,0143$ & $-0,0578$ & $-0,0628$ \\
LEITE FLUIDO & $-0,0149$ & 0,0136 & 0,0085 & $-0,0046$ & $-0,0538$ & $-0,0284$ \\
MACARRÃO & $-0,0129$ & $-0,0010$ & 0,0102 & 0,0070 & 0,0065 & 0,0061 \\
MANTEIGA & $-0,0566$ & $-0,0101$ & 0,0058 & $-0,0006$ & $-0,0361$ & $-0,0270$ \\
MARGARINA & $-0,1283$ & $-0,0564$ & $-0,0073$ & 0,0262 & $-0,0418$ & $-0,0397$ \\
PAO FRANCÊS & $-0,0301$ & 0,0033 & $-0,0032$ & $-0,0167$ & $-0,0516$ & $-0,0539$ \\
SUÍNO & $-0,0829$ & $-0,0457$ & 0,0097 & 0,0333 & 0,0468 & 0,0665 \\
QUEIJOS & $-0,0396$ & $-0,0093$ & 0,0105 & $-0,0104$ & $-0,0243$ & $-0,0432$ \\
\hline TOMATE & & & & &
\end{tabular}

Fonte: Dados da Pesquisa.

Tabela 8. Efeitos marginais das variáveis de raça, Brasil, Período 2002 - 2003

\begin{tabular}{lcccc}
\hline \multicolumn{1}{c}{ PRODUTOS } & NEGRA & AMARELA & PARDA & OUTRA \\
\hline AÇÚCAR & 0,0133 & $-0,0319$ & 0,0222 & $-0,0472$ \\
ARROZ & 0,0198 & 0,0230 & 0,0255 & $-0,0846$ \\
BANANA & $-0,0531$ & $-0,0306$ & $-0,0190$ & $-0,0321$ \\
BATATA & $-0,0411$ & $-0,0109$ & $-0,0149$ & $-0,0325$ \\
BOIPRIMEIRA & $-0,0605$ & 0,0649 & $-0,0238$ & $-0,0166$ \\
BOISEGUNDA & 0,0335 & 0,0424 & 0,0186 & $-0,0310$ \\
FARINHA DE & 0,0460 & 0,0045 & 0,0244 & 0,0010 \\
MANDIOCA & & & & \\
FEIJÃO & 0,0041 & $-0,0513$ & 0,0172 & $-0,0856$ \\
FRANGO & 0,0221 & $-0,0182$ & 0,0119 & $-0,0715$ \\
LEITE EM PÓ & 0,0270 & $-0,0083$ & 0,0179 & $-0,0156$ \\
LEITE FLUIDO & $-0,0806$ & $-0,1209$ & $-0,0586$ & $-0,1233$ \\
\hline
\end{tabular}


Tabela 8. Continuação.

\begin{tabular}{lcccc}
\hline \multicolumn{1}{c}{ PRODUTOS } & NEGRA & AMARELA & PARDA & OUTRA \\
\hline MACARRÃO & $-0,0247$ & $-0,0336$ & 0,0010 & $-0,0464$ \\
MANTEIGA & 0,0084 & $-0,0134$ & 0,0036 & 0,0003 \\
MARGARINA & $-0,0105$ & $-0,0796$ & $-0,0041$ & $-0,0660$ \\
PAO FRANCÊS & $-0,0194$ & 0,0084 & $-0,0036$ & $-0,0792$ \\
SUÍNO & 0,0162 & 0,0382 & 0,0090 & $-0,0562$ \\
QUEIJOS & $-0,0424$ & $-0,0581$ & $-0,0456$ & $-0,0766$ \\
TOMATE & $-0,0233$ & 0,0784 & $-0,0029$ & $-0,0622$ \\
\hline
\end{tabular}

Fonte: Dados da Pesquisa.

Para o caso da variável "mulher" (responsável pelo domićlio ser do sexo feminino) (Tabela 9), de forma geral, os efeitos marginais têm magnitudes menores que as demais variáveis. Destacam-se o impacto negativo nas probabilidades de aquisição de arroz (-5,76 p.p.) e feijão (-5,56 p.p.), assim como as carnes em geral. Esse resultado parece indicar uma menor probabilidade de refeições feitas no domicílio quando a mulher é a responsável. Certamente, a dificuldade do preparo das refeições pela mulher que trabalha fora impõe a substituição das refeições no domicílio por alternativas fora dele ${ }^{16}$.

Em relação à variável "doméstica" (presença de empregada doméstica no domicílio) (Tabela 10), as magnitudes são comparáveis à variável "mulher". Os destaques são os impactos positivos na probabilidade de aquisição de carne bovina de primeira (4,18 p.p.) e banana (2,98 p.p.) e negativos nas probabilidades para farinha de mandioca (-3,95 p.p) e carne de boi de segunda (-3,01 p.p.). De forma geral, os resultados mostram um impacto negativo em produtos básicos (arroz, feijão, farinha de mandioca) e positivo em produtos mais "nobres", como queijos e carnes de primeira. A expectativa inicial, como já colocado no item anterior, era de um impacto positivo da presença de empregada doméstica na realização de refeições no domićlíio e, assim, também nos produtos mais utilizados, como arroz e feijão. Os resultados, entretanto, não confirmaram esta expectativa. Mesmo controlando para a renda, a presença de empregada doméstica parece permitir uma diferenciação entre domicílios que favorece o consumo de alimentos mais caros, diminuindo a probabilidade de aquisição para os básicos.

\footnotetext{
${ }^{16}$ No caso das crianças, refeições em creches e escolas podem substituir parte daquelas realizadas em casa. Para os adultos, refeições no local de trabalho ou em estabelecimentos próximos podem ser substitutos escolhidos.
} 
Tabela 9. Efeitos marginais da variável "mulher", Brasil, Período $2002-2003$

\begin{tabular}{lc}
\hline PRODUTOS & EFEITO MARGINAL \\
\hline AÇÚCAR & $-0,0337$ \\
ARROZ & $-0,0576$ \\
BANANA & 0,0102 \\
BATATA & $-0,0034$ \\
BOIPRIMEIRA & $-0,0107$ \\
BOISEGUNDA & $-0,0228$ \\
FARINHA DE MANDIOCA & $-0,0245$ \\
FEIJÃO & $-0,0556$ \\
FRANGO & $-0,0157$ \\
LEITE EM PÓ & 0,0015 \\
LEITE FLUIDO & $-0,0027$ \\
MACARRÃO & $-0,0258$ \\
MANTEIGA & 0,0027 \\
MARGARINA & $-0,0001$ \\
PAO FRANCÊS & 0,0147 \\
SUÍNO & $-0,0244$ \\
QUEIJOS & 0,0147 \\
TOMATE & $-0,0184$ \\
\hline
\end{tabular}

Fonte: Dados da Pesquisa.

Finalmente, em relação à variável "geladeira" (presença de geladeira no domicílio) (Tabela 11), destaque para o impacto na probabilidade de aquisição de pão francês (13,12 p.p.) e leite fluido (10,64 p.p.). As carnes em geral (com exceção da carne suína) e queijos também apresentam impactos positivos importantes. A probabilidade de aquisição de leite em pó é afetada negativamente (-1,56 p.p.), mas numa magnitude menor que a esperada. A conclusão no caso do leite parece ser que, dada a necessidade de refrigeração, o leite fluido é afetado de forma mais significativa e a substituição pelo leite em pó é realizada também por outras razões não-relacionadas apenas com a refrigeração ${ }^{17}$.

\footnotetext{
${ }^{17}$ Aspectos como comodidade, diferenciação de produto, presença de características especiais devem influenciar também o consumo de leite em pó.
} 
Padrão de consumo de alimentos no Brasil

Tabela 10. Efeitos marginais da variável "doméstica", Brasil, Período 2002 - 2003

\begin{tabular}{lc}
\hline PRODUTOS & EFEITO MARGINAL \\
\hline AÇÚCAR & $-0,0164$ \\
ARROZ & $-0,0031$ \\
BANANA & 0,0298 \\
BATATA & 0,0245 \\
BOIPRIMEIRA & 0,0418 \\
BOISEGUNDA & $-0,0301$ \\
FARINHA DE MANDIOCA & $-0,0395$ \\
FEIJÃO & $-0,0160$ \\
FRANGO & $-0,0201$ \\
LEITE EM PÓ & 0,0002 \\
LEITE FLUIDO & 0,0199 \\
MACARRÃO & $-0,0061$ \\
MANTEIGA & 0,0096 \\
MARGARINA & $-0,0236$ \\
PAO FRANCÊS & $-0,0227$ \\
SUÍNO & $-0,0140$ \\
QUEIJOS & 0,0190 \\
TOMATE & 0,0217 \\
\hline
\end{tabular}

Fonte: Dados da Pesquisa

Tabela 11. Efeitos marginais da variável "geladeira”, Brasil, Período 2002 - 2003

\begin{tabular}{lc}
\hline PRODUTOS & EFEITO MARGINAL \\
\hline AÇÚCAR & $-0,0434$ \\
ARROZ & $-0,0515$ \\
BANANA & 0,0436 \\
BATATA & 0,0699 \\
BOIPRIMEIRA & 0,0629 \\
BOISEGUNDA & 0,0354 \\
FARINHA DE MANDIOCA & $-0,0467$ \\
FEIJÃO & $-0,0329$ \\
FRANGO & 0,0441 \\
LEITE EM PÓ & $-0,0156$ \\
LEITE FLUIDO & 0,1064 \\
MACARRÃO & 0,0185 \\
\hline
\end{tabular}


Tabela 11. Continuação.

\begin{tabular}{lc}
\hline PRODUTOS & EFEITO MARGINAL \\
\hline MANTEIGA & 0,0122 \\
MARGARINA & 0,0535 \\
PAO FRANCÊS & 0,1312 \\
SUÍNO & $-0,0052$ \\
QUEIJOS & 0,0653 \\
TOMATE & 0,0717 \\
\hline
\end{tabular}

Fonte: Dados da Pesquisa.

\section{Conclusão e implicações}

Os principais resultados deste estudo permitem as seguintes conclusões:

- O efeito da variável renda familiar mensal na probabilidade de aquisição é negativo para os produtos básicos e positivos para os demais produtos;

- Há diferenças regionais significativas na probabilidade de consumo entre as regiões do País, mesmo controlando para as demais variáveis.

- Há diferenças, também, entre domicílios localizados nos meios urbano e rural. Na zona rural, há maior probabilidade de aquisição de produtos básicos, mais energéticos, e também maior probabilidade de consumo de produtos em que há possibilidade de produção própria, como o leite fluido. No meio urbano, destaca-se a maior probabilidade de aquisição de pão francês.

- O nível educacional parece estar negativamente correlacionado com a probabilidade de aquisição dos produtos da cesta pesquisada, especialmente para os produtos básicos.

- Há diferenças significativas nas probabilidades de aquisição entre domicílios chefiados por pessoas que se declararam pardas e negras e os domicílios chefiados por brancos. Essa diferença está na maior probabilidade de consumo de produtos básicos e carne bovina de segunda para domicílios chefiados por pardos e negros e maior probabilidade de aquisição de produtos como carne bovina de primeira, banana, queijos e leite fluido para domicílios chefiados por brancos.

- Domicílios chefiados por mulheres têm menor probabilidade de aquisição para consumo domiciliar de quase todos os produtos da cesta escolhida.

- A presença de empregada doméstica no domicílio teve efeito oposto ao esperado, com um impacto negativo na probabilidade de aquisição de produtos básicos (arroz, feijão, farinha de mandioca). 
- A presença de geladeira no domicílio permite aos domicílios substituir parte dos produtos básicos por aqueles que necessitam de refrigeração, com destaque para o leite fluido.

Esses resultados trazem diversas implicações, tanto para estratégias empresariais quanto para o poder público. Para o investidor privado, é importante saber que mudanças no padrão de renda da população tendem a afetar de forma diversa os diferentes tipos de produtos. Os resultados mostram, por exemplo, que o aumento da renda tende a elevar o consumo domiciliar de produtos como queijos e carne bovina de primeira e diminuir o consumo de produtos básicos, como arroz e feijão. Essa informação é importante para o setor de varejo, que pode ajustar seu conjunto de produtos vendidos de acordo com a resposta dos consumidores. O conhecimento das diferenças de comportamento entre níveis de escolaridade, raça, e gênero do comprador é também de grande relevância para o planejamento das estratégias de marketing para os gêneros alimentícios. No tocante ao governo, o conhecimento do padrão de demanda permite antecipar os efeitos de choques de oferta para as diferentes regióes e para os diferentes grupos de consumidores, indicando políticas que previnam seus efeitos negativos. Além disso, permite projetar como os programas de complementação de renda irão influenciar no padrão alimentar e conseqüente condição nutricional da população atendida. Por exemplo, os resultados indicam um maior consumo de proteína animal (carnes, leite e queijos) e menor consumo de carboidratos (arroz, farinha de mandioca) com um aumento da renda.

Finalizando, é importante ressaltar algumas limitações do trabalho, tal como o pequeno número de frutas e verduras entre os produtos estudados e o fato de o estudo não investigar as aquisições alimentares fora do domicílio. Este último fato deve-se à limitação da base de dados do IBGE, que não permite uma desagregação entre os alimentos consumidos fora do domicílio. Quanto às frutas e verduras, são necessários ainda mais estudos que investiguem a hipótese de maior preocupação dos consumidores com o consumo de alimentos mais saudáveis e nutritivos.

\section{Referências Bibliográficas}

AGUERO, J. M.; GOULD, B. W. Household Composition and Brazilian Food Purchases: An Expenditure System Approach. Canadian Journal of Agriculture Economics, Vol.51, n. 3, p. 323-345, 2003.

BACCHI, M.R.P. Demanda de carne bovina no mercado brasileiro. Piracicaba, 1989, ESALQ/USP, 77p. (tese de mestrado). 
BRANDT, S.A.; AAD NETO, A.; REZENDE, A. M.; SOUZA, A. F.; SOUZA, D.C. Análise econômica e projeções da demanda de carne bovina no mercado de Manaus. Estudos de Economia Agrícola, Manaus, ACAR, 106p, 1973.

DAVIDSON, R.; MACKINNON, J. Estimation and inference in econometrics. New York, Edição única. Oxford University Press, 1993, 874p.

DEATON, A. \& MUELLBAUER J. Economics and Consumer Behavior. New York: Cambridge University Press, 1980, 450 p.

FIALLOS, L.E.W. Análise da demanda e preços de tomate no estado de São Paulo. Piracicaba, 1982, ESALQ/USP, 116p. (tese de mestrado).

FURTUOSO, M. C. O. Redistribuição de e consumo de Alimentos no Estado de São Paulo. Piracicaba, 1981, ESALQ/USP, 106p. (tese de mestrado).

GREENE, W.H. Econometric Analysis. Prentice Hall, Upper River,4 edition, 2000, 1004p.

GRIFFITHS, W.E; HILL, R.C.; JUDGE, G.G. Learning and practicing econometrics. New York: John Wiley \& Sons, 1993, 866p.

HAINES, P. S., GUILKEY, D. K., POPKIN, B.M. Modeling Food Consumption Decisions as a Two-Step Process. American Journal of Agricultural Economics, Vol. 70, No. 3 (Aug., 1988) , pp. 543-552

HOFFMANN, R. A diminuição do consumo de feijão no Brasil. Estudos Econômicos, Vol. 25, n. 2, p. 189-201, Maio-Ago, 1995.

Elasticidades-renda das despesas e do consumo físico de alimentos no Brasil metropolitano em 1995-96. Agricultura em São Paulo, SP, v.47, n.1 p. 111-122, 2000.

IBGE. Pesquisa de Orçamentos Familiares 2002-2003. Aquisição alimentar domiciliar per capita: Brasil e Grandes Regiões. Rio de Janeiro: Instituto Brasileiro de Geografia e Estatística, 2004a.

IBGE. Pesquisa de Orçamentos Familiares 2002-2003. Primeiros Resultados: Brasil e Grandes Regiões. Rio de Janeiro: Instituto Brasileiro de Geografia e Estatística, 2a edição, 2004b.

IBGE. Pesquisa de Orçamentos Familiares 2002-2003. CD-ROM - Microdados Segunda divulgação. Rio de Janeiro: Instituto Brasileiro de Geografia e Estatística, 2004c.

MUSGROVE, P. Family Expenditure and Food prices as determinants of Food consumption in Northeast Brazil. Washington D.C., Pan American Health Organization, 1986. 
SHONKWILER, J.S. \&. YEN, S.T. Two-Step Estimation of a Censored System of Equations. American Journal of Agricultural Economics. Vol. 81, n.4, 972-982, Nov. 1999.

THOMAS, D., STRAUSS, J., BARBOSA, M. M. T. Estimativas do impacto de mudanças de renda e de preços no consumo no Brasil. Pesquisa e Planejamento Econômico, v.21, n.2, p.305-354, 1991. 